\title{
Publicaciones para inmigrantes: estudio de las informaciones y la publicidad dirigidas a la comunidad inmigrante
}

Publications for Immigrants: Study of the Information and

\section{Advertisement Targeting the Immigrant Community}

Esther Martínez Pastor. Universidad Rey Juan Carlos

Marina Santín Durán. Universidad Rey Juan Carlos

Recibido: 27-II-2009 - Aceptado: 26-VI-2009

Resumen:

En los últimos años se asiste a la multiplicación de medios de comunicación que tienen como principal publico objetivo a la población extranjera. Este artículo da a conocer los resultados de dos investigaciones que se centraron en el estudio de los mensajes publicitarios y de las informaciones que aparecen en esos medios. Dicho análisis nos lleva a concluir que los medios para los inmigrantes contribuyen a la cohesión cultural de sus receptores, les permiten recrear su propia cultura y sus valores y al mismo tiempo les permiten acceder a una información hecha a su medida, ajustada a sus necesidades y desde su propia visión.

Palabras clave:

Inmigración, identidad, medios impresos, periodismo y publicidad.

Summary:

In the most recent years we are witnessing an increase in mass media that is directed mainly to the foreign population. This article showcases the results obtained from two independent research projects which focused on advertisement and information shown in those media targeting immigrants. Data obtained from these studies allow us to conclude that media targeting immigrants contribute to their cultural cohesion, allow the immigrants to recreate their own culture and values, and at the same time allow them access to information made-to-order, adjusted to their needs, and from their own perspective.

Key Words:

Immigration, Identity, Mass Media, Journalism and Advertising. 


\section{Introducción}

La sociedad española ha experimentado en los últimos diez años un gran cambio fruto de los movimientos migratorios. España, que durante décadas fue punto de partida de millones de personas que iban al norte de Europa o cruzaban el Atlántico "haciendo las Américas", es hoy una gran receptora de población inmigrante. Según los datos del INE del 2008 un 11,33\% de los ciudadanos que residen en España son de origen extranjero. La presencia de esta población inmigrante genera una diversidad socio-cultural que tiene un claro reflejo en diversos ámbitos de la comunicación. Y es que ciudadanos ecuatorianos, colombianos, rumanos, chinos, marroquíes, entre otros, en España, protagonizan noticias y también anuncios que muchas veces se dirigen casi exclusivamente a ellos.

El progresivo aumento de la población inmigrante ha generado, tal y como ha pasado en otros países con mayor tradición multicultural, como Estados Unidos o Reino Unido, un gran número de medios de comunicación étnicos o medios de la diáspora, que son un fenómeno comunicativo novedoso en España que requiere ser analizado en profundidad.

Este artículo da a conocer las principales conclusiones a las que llegaron dos trabajos paralelos y complementarios sobre los medios de la inmigración en España que se han desarrollado en la Universidad Rey Juan Carlos y que analizan las informaciones y la publicidad difundida en esos medios. El primero de ellos fue un proyecto de investigación ${ }^{1}$ financiado por la Comunidad de Madrid y la Universidad Rey Juan Carlos para el estudio de los medios de la inmigración que se centró en los contenidos informativos y la labor de servicio que estos medios pueden desempeñar. El segundo trabajo está centrado en la publicación "La Publicidad se acerca a la inmigración” (VVAA: 2007)² y en particular en el capítulo titulado "Campañas publicitarias para ciudadanos inmigrantes”, de Martínez Pastor, así como en el resultado de las II Jornadas de "Publicidad e Inmigración" celebradas en 2008. En dicho capítulo y en las jornadas se identifican dos categorías de publicidad: "las campañas integradoras" y "las campañas con el ciudadano inmigrante como target”. En las primeras el objetivo es dual: de un lado, sensibilizar a los autóctonos de la llegada de los inmigrantes y, de otro lado, integrar a éstos en nuestra sociedad, cuyos anunciantes son principalmente las Administraciones Públicas, las fundaciones, las ONG’s y las asociaciones sin ánimo lucrativo. Mientras que "las campañas con el ciudadano inmigrante" constan de dos subcategorías: las campañas ethnic (enfoca-

\footnotetext{
“Diversidad cultural en Madrid: medios de comunicación de la comunidad inmigrante" (Programa de Creación y consolidación de grupos de investigación- Referencia: URJC-CM-2006-CSH-0304) Investigadora principal: Gloria Gómez-Escalonilla.

2 Esta publicación es fruto de los resultados obtenidos de la investigación "Proyección de la diversidad cultural y de la integración de la inmigración en la Región de Murcia a través de la publicidad” subvencionado por la Consejería de Trabajo y Política Social de la Región de Murcia.
} 
das exclusivamente para los inmigrantes) y las campañas híbridas (dirigidas tanto a nacionales como a inmigrantes con fin lucrativo). Desde este punto de vista la investigación relativa a publicidad parte de las campañas ethnic en medios impresos para conocer quién y qué se anuncia en dichos medios.

Precisamente este es el punto en el que se enlazan las dos investigaciones. Ambas pretenden conocer al colectivo de los inmigrantes a través de los medios impresos. Saber qué se les está ofreciendo desde un punto de vista informativo y publicitario a los inmigrantes y si esos mensajes representan sus necesidades y deseos. Y, asimismo, reflexionar si los contenidos informativos y los publicitarios, al unísono, reflejan la misma realidad del colectivo de inmigrantes.

\section{Los medios de la inmigración: ¿ Un fenómeno nuevo?}

Los medios de comunicación especializados en el colectivo inmigrante también son llamados medios de la diáspora. El término diáspora en este contexto pretende poner de relieve el sentido de pertenencia a una comunidad más allá de los límites espaciales de una nación. Se refiere a comunidad que comparte unos valores culturales y lingüísticos e incluye a las personas que han experimentado la migración o que son hijos de inmigrantes.

Si bien estos medios de la diáspora son una novedad en las ciudades españolas, lo cierto es que en otros países son medios consolidados. En el mercado de la prensa norteamericana se encuentran una gran diversidad de medios destinados a inmigrantes; algunos de ellos tienen una gran trayectoria a sus espaldas y presentan altos índices de audiencia. A modo de ejemplo podemos señalar el periódico La Prensa (más tarde Diario-La Prensa) de Nueva York presente desde 1913 y dirigido específicamente a la comunidad latinoamericana allí asentada. En Europa, en países como el Reino Unido, Francia o Alemania se editan desde hace décadas medios de comunicación que se dirigen a la comunidad migratoria, principalmente a la comunidad árabe, a las minorías asiáticas e indias o los inmigrantes que vienen de países Orientales europeos. Por ejemplo: India Weekly, Asian Times, Arabic- Al-Diplomasi News Report en Reino Unido; Daily Ausaf, Nouvelles d'Europe y Turkish News en Alemania; Al Mustakbal en Francia.

Si bien en España los estudios sobre estos medios ${ }^{3}$ son tan recientes como el propio fenómeno, en otros lugares, por ejemplo en Estados Unidos, son muchos los trabajos desarrollados sobre estos medios tanto en su aspecto informativo como publicitario.

3 Los más destacables son Lorite (2004), Gómez Escalonilla (2007) y Retis (2008) entre otros. 
Desde una perspectiva periodística hay una línea asentada en los estudios académicos norteamericanos, donde destacan las investigaciones del Pew Hispanic Center con la Universidad del Sur de California (Suro, 2004) o por Rojas (2004), Rios, D. y Mohamed (Eds.) (2003) o Benítez (2007) y la Asociación Nacional de Periodistas Hispanos. Desde el ámbito académico español también se han realizado trabajos de investigación que tomaron como objeto de estudio los medios étnicos norteamericanos 4 . En el entorno europeo los proyectos más destacables son Diasporic Minorities and their Media in the EU: a mapping, del European Media Technology, dirigido por M. Georgiou y Minority Media: Minority, Media and Representation across Europe, de la Universidad de Poitiers, dirigido por Isabell Rigoni.

Desde la perspectiva publicitaria, los estudios norteamericanos desde los años 60 se centran en dos líneas diferenciadas: una aborda la necesidad de segmentar al colectivo de inmigrantes porque proviene de culturas diferentes; otra considera que es absurdo hacer diferentes tipos de publicidad porque no hay datos reales de un consumo diferente entre los distintos colectivos de inmigrantes. La primera línea de investigación está amparada en la teoría de la accomodation intercultural cuyos investigadores consideran que los anunciantes deben dirigir sus mensajes publicitarios de forma distinta a cada colectivo de inmigrantes para conseguir mayor eficacia (Lee, Fairhurst y Dillard: 2002, 27). Esta teoría afirma que los inmigrantes vienen con su propia cultura de origen y al instalarse en el país de destino se sumergen en la cultura dominante despojándose un poco de la propia y adaptándose a la ajena (Hollard y Gentry, 1999: 68). Por ello, en este proceso de "acomodación" es aconsejable que la publicidad les recuerde sus orígenes a través de su idioma materno y de sus símbolos para conseguir su atención como consumidores (Torres y Gelb, 2002: 69).

Sin embargo y a pesar de esta teoría, lo cierto es que en Estados Unidos hay numerosos medios de comunicación y anuncios segmentados según los diferentes colectivos de inmigrantes con un éxito incuestionable.

\section{Metodología y objeto de estudio}

Los resultados que aquí se presentan son fruto de dos proyectos de investigación que se desarrollaron de manera independiente y cada uno tuvo una especificidad propia en marcos analíticos diferentes. Uno se centra en el mensaje periodístico y el otro en los mensajes publicitarios. Sin embargo, sendos estudios se complementan porque comparten el mismo objeto de estudio, la inmigración y los medios impresos en la Comunidad de Madrid, y una misma metodología de estudio.

4 Mompart (2008) sobre la prensa en español de Nueva York y los trabajos realizados por los investigadores Xavier López y Rosa Franquet, Francesc Ribes, Emili Prado y Matilde Delgado (2004) sobre las industrias culturales en español en Estados Unidos que han sido publicados en la revista Telos. 
El objetivo de las dos investigaciones perseguía comprobar si las publicaciones y los contenidos informativos y publicitarios dirigidos al colectivo de inmigrantes satisfacen las necesidades de la comunidad inmigrante y cumplen con la función social de ser mediadores socioculturales y comunicacionales. Y así, conocer sus hábitos de vida e intereses como consumidores mediáticos.

Dichas investigaciones comparten la misma metodología: primero, se han identificado los diarios más leídos por la población inmigrante en la Comunidad Autónoma de Madrid; posteriormente, se ha elegido el lapso de tiempo para llevar a cabo el estudio y, finalmente, se ha realizado el análisis de los datos obtenidos.

Las diferencias entre ambas investigaciones residen en los aspectos cuantitativos de la selección de la muestra, el lapso de tiempo de análisis y, por supuesto, el punto de vista del análisis periodístico y publicitario. Pero, como hemos indicado anteriormente, y a pesar de estas diferencias, los resultados se complementan para estudiar el papel de los medios impresos y el colectivo de los inmigrantes.

Estas investigaciones acotaron el estudio a las publicaciones más leídas por los inmigrantes con mayor presencia y mayor difusión, a saber: Latino y Sí, se puede, Ocio Latino, Tümai, 20 Minutos, Metro, El País, El Mundo. Tanto los resultados del EMI (Estudios de Medios para la inmigración) ${ }^{5}$ como los datos ofrecidos por la PGD (Control de Publicaciones de Distribución Gratuita), división de la OJD (Oficina de Justificación de Datos), certifican que estas son las publicaciones más leídas. La publicación mensual que ha sido excluida de esta investigación ha sido Raíz pues presenta doce cabeceras diferentes (Raíz Africana, Raíz Argentina, Raíz Bolivia, Raíz Bulgaria, Raíz Colombia, Raíz Dominicana, Raíz Ecuador, Raíz Marruecos...) segmentadas por colectivos aunque se compute como una única publicación a efectos de difusión.

En el caso del estudio de los contenidos informativos, éste se centró en el segmento de la comunidad latina con las cabeceras Latino y Sí, se puede, si bien el estudio de publicidad analizaba todas las publicaciones diarias, semanales y mensuales. La muestra obtenida en el estudio de prensa es de 800 unidades informativas, 400 por cada semanario, y la muestra publicitaria de 221 anuncios. Las cabeceras analizadas en ambos casos son Latino y Sí, se puede y, como se explicará más adelante, los anuncios publicitarios se repiten en el resto de los diarios con lo cual, en este sentido, sendas investigaciones se complementan.

El lapso de tiempo seleccionado es del periodo comprendido de julio de 2007 a junio de 2008, en la investigación de los contenidos periodísticos, y en el de publicidad de enero a marzo de 2008. Metodológicamente el periodo temporal de análisis en ambas investigaciones coincide en estos últimos meses.

5 El EMI es uno de los estudios pioneros en conocer los hábitos de consumo mediático del colectivos de los inmigrantes. El universo seleccionado para la investigación fue limitado a la Comunidad de Madrid a través de 1919 entrevistas durante el 2007. 
El estudio relativo a los mensajes periodísticos de ambos ítems se llevó a cabo con la técnica del análisis de contenido relativo a la temática, ámbitos de las noticias y sección de la misma, entre otros con el propósito de conocer si la prensa cubre las necesidades del colectivo inmigrante. Y complementando esta información, el estudio del análisis publicitario ha llevado a cabo una radiografía de la oferta de productos y servicios dirigidos al target de inmigrantes en los medios impresos más leídos y difundidos con el propósito de conocer qué les están ofertando los anunciantes y, así, conocer los hábitos de vida de los consumidores a través de los anuncios.

Una vez explicados en líneas generales la muestra, el periodo de tiempo analizado y el estudio relativo a los mensajes de contenidos y publicitarios, pasamos a detallar la selección de la muestra en cada una de las investigaciones y los puntos en común que hemos detectado para poder reflexionar sobre el papel de los medios y los inmigrantes. La selección de la muestra en ambas investigaciones responde a los siguientes criterios. En cuanto a los diarios semanales Latino y Sí, se puede son los periódicos más difundidos a nivel nacional y con mayor número de lectores en la Comunidad de Madrid, según la PGD y la EMI. Latino cuenta con una difusión nacional de 140.656 ejemplares y con un número de lectores en Madrid de 196.6000 por semana y Sí, se puede tiene una difusión de 175.922 ejemplares y 120.200 lectores por semana.

En los diarios mensuales hemos seleccionado Ocio Latino y Tümai cruzando los datos ofrecidos por PGD y EMI durante el 2007. Conviene señalar que la PGD durante el año 2006 destaca como el diario más leído Raíz seguido de Cultures Romania. Sin embargo hemos descartado estos diarios para esta muestra primero porque estos datos son del 2006 y para nuestros estudio nos interesaban datos más recientes y, en segundo lugar, porque en el caso del diario Raíz está editado en varios idiomas y la PGD no especifica cual de los idiomas es el de mayor difusión, simplemente los computa de forma general, como anteriormente dijimos. Y en relación con Cultures Romania, la EMI no lo contempla como diario más leído en la Comunidad de Madrid. Por este motivo cruzando los datos más actuales y las publicaciones mensuales más leídas en este territorio optamos por Ocio Latino, con 30.320 ejemplares difundidos y 120.200 lectores por mes, y Tümai, con una difusión de 77.502 ejemplares y 35.000 lectores mensuales en Madrid.

En cuanto a los diarios de información general gratuitos leídos por los inmigrantes encontramos datos muy distintos en la PGD y la EMI. La PGD indica que los diarios más difundidos son 20 Minutos y Qué mientras que según la EMI se sitúan en primer lugar Metro seguido de 20 Minutos. En este caso para seleccionar los diarios más adecuados a nuestro estudio optamos por 20 Minutos, diario en el que ambas entidades coincidían, y por Metro dado que es el diario más leído en Madrid, territorio en el que se centra el presente estudio. 20 Minutos cuenta con una difusión de 1.042.254 y con 220.700 lectores/ día y Metro con una difusión de 840.259 ejemplares y 226.500 lectores/día. 
Finalmente, seleccionamos El País (tirada 560.176 y 62.100 lectores/día) y El Mundo (tirada 441.26 .800 lectores/día) como los diarios de información general de pago más difundidos y con mayor número de lectores tanto según la OJD como el EMI. [Véase a continuación la tabla que resume el corpus analizado en las investigaciones].

Tabla I. Objeto de estudio

\begin{tabular}{|c|c|c|c|}
\hline Investigaciones & Objeto de la muestra & Muestra & Periodo temporal \\
\hline Análisis periodístico & $\begin{array}{c}800 \text { unidades / } \\
\text { informativas } \\
400 \text { por cada } \\
\text { semanario }\end{array}$ & $\begin{array}{c}\text { Latino } \\
\text { (140.656 difusión nacional / } \\
\text { 196, } 600 \text { lectores/semana) } \\
\text { Sí, se puede } \\
\text { (175.922 difusión nacional / } \\
\text { 120,200 lectores/semana) }\end{array}$ & Julio-2007- junio 2008 \\
\hline Análisis publicitario & 221 anuncios & $\begin{array}{c}\text { Latino } \\
\text { (140.656 difusión nacional / } \\
\text { 196, } 600 \text { lectores/semana) } \\
\text { Sí, se puede } \\
\text { (175.922 difusión nacional / } \\
\text { 120,200 lectores/semana) } \\
\text { Ocio Latino } \\
\text { (30.320 difusión nacional / } \\
41,800 \text { lectores/mes) } \\
\text { Tümai } \\
\text { (77.502 difusión nacional/ } \\
35.000 \text { lectores/mes) } \\
20 \text { Minutos } \\
\text { (1.042.254 difusión nacional/ } \\
220,700 \text { lectores/día) } \\
\text { Metro } \\
\text { (840.259 difusión nacional/ } \\
\text { 226,500 lectores/día) } \\
\text { El País } \\
\text { (560.176 difusión nacional/ } \\
62,100 \text { lectores/día) } \\
\text { El Mundo } \\
\text { (441.880 difusión nacional/ } \\
\text { 26,800 lectores/día) }\end{array}$ & Enero-marzo de 2008 \\
\hline
\end{tabular}

Fuente: PGD, EMI Y OJD y elaboración propia 
Explicado el objeto de la muestra y la metodología pasamos a detallar los resultados de las investigaciones y los puntos en común de ambos estudios, como anteriormente se ha expuesto.

\section{Resultados}

\subsection{Periodismo para inmigrantes}

Diversos estudios académicos han mostrado cómo los medios de comunicación han desempeñado un papel central en la reproducción de un discurso adverso sobre los inmigrantes centrado en "resaltar los problemas que causan en la sociedad de acogida o las dificultades que encuentran para su aceptación" (Granados, 2006: 60). A esa representación de la inmigración llena de estereotipos se suma el hecho de que los medios tradicionales no suelen tampoco responder a las necesidades de información de los nuevos ciudadanos que requieren, con frecuencia, de una información útil y práctica sobre el país de acogida.

La necesidad de un cambio en los medios dominantes es requisito indispensable para que desde ese cuarto poder se contribuya a la integración social del inmigrante y a favorecer la vida cotidiana de los recién llegados. Para alcanzar ese logro han surgido numerosos manuales que recomiendan a los periodistas cómo llevar a cabo el tratamiento del fenómeno migratorio. Uno de los primeros fue el Manual de estilo sobre tratamiento de las minorías étnicas en los medios de comunicación social que en 1996 publicó el Colegio de Periodistas de Cataluña ${ }^{6}$.

A la espera de un tratamiento informativo más próximo a los intereses de las comunidades inmigrantes nacen los medios de la diáspora como Latino y Sí, se puede en los que se centra esta investigación.

Ambas publicaciones se caracterizan por la presencia de textos breves que casi siempre se acompañan de algún elemento gráfico. Son periódicos en los que el color juega un papel muy importante en el diseño de sus informaciones. Y éstas no suelen firmarse pues de cada diez textos informativos sólo en dos de ellos figura la firma del periodista que se ha hecho cargo de la información. Posiblemente el hecho de que muchos

6 Las principales recomendaciones que da son: No "estigmatizar" a la persona o al grupo étnico del que se está informando, haciendo referencia innecesaria a su grupo étnico, el país de origen, su religión o cultura. Rechazar las generalidades y la simplificación sobre las personas inmigrantes, teniendo en cuenta que los residentes extranjeros son tan poco homogéneos como los autóctonos. Evitar las informaciones negativas y sensacionalistas, potenciando las informaciones positivas. Dejar oír la voz de los propios inmigrantes. No buscar exclusivamente la "fiabilidad" de la fuente institucionalizada, como Administración, fuerzas de seguridad u ONGs, dejando al margen la información que puedan ofrecer las asociaciones y los colectivos de inmigrantes. Apelar a la responsabilidad de los profesionales en el tratamiento y el formato de las informaciones. 
textos sean muy breves y que los redactores se hagan cargo semanalmente de varios artículos conlleva que muchos de ellos no se firmen para no evidenciar esa situación.

Los medios étnicos, medios de la inmigración, aseguran intentar dar respuesta a las demandas informativas de los nuevos residentes y favorecer el proceso de integración de los mismos. Con frecuencia el ciudadano inmigrante que llega a España añora su país y desea saber de los suyos pero también necesita de información que le ayude a descubrir su nuevo país. Esas dos demandas o necesidades informativas conviven de forma clara en los medios analizados. Son periódicos que centran sus contenidos en la promoción de valores autóctonos y que ofrecen una información de proximidad, servicio y utilidad para el lector.

\subsubsection{Medios locales en un contexto internacional}

Las publicaciones analizadas se caracterizan por el hecho de informar con frecuencia de temas actuales del país de acogida. Las informaciones locales y nacionales tienen una presencia destacable en los medios analizados pues más de la mitad de los textos informativos que aparecen en esas publicaciones hacen referencia a hechos que suceden en territorio nacional. España es el lugar que más informaciones genera para estos diarios. El 31,9\% de las informaciones suceden en el ámbito nacional y el 26,2\% en el local.

La información nacional presenta la peculiaridad de tener un enfoque próximo a los intereses de los inmigrantes. Que la noticia se desarrolle en España no conlleva necesariamente que aborde un tema o asunto exclusivo de la nación de acogida. De hecho, resulta relativamente frecuente que esas informaciones toquen temas relativos a los países que más inmigrantes aportan a España o que sean noticias protagonizadas por gentes de esos países. De este modo, la información nacional muestra en estos medios ciertas peculiaridades que la diferencian de la información nacional de los medios dominantes.

La información local o autonómica representa una de cada cuatro informaciones en los medios analizados. Esa información, que geográficamente es la más próxima, no sólo está presente en la sección destinada a la misma, -Madrid Latino en Latino y En tu comunidad en Sí, se puede-, sino que también tiene gran presencia en otras secciones como, por ejemplo, Deportes o Servicios. El rasgo que más caracteriza a la información local, además de ser de proximidad, es el de ser una información de servicio.

La información internacional, la que sucede fuera de nuestras fronteras, también está presente en estas publicaciones con un toque especial. Todos los medios suelen ofrecer noticias alusivas a terceros países pero no todos prestan la misma atención a unos que a otros. De hecho en los medios de la inmigración los países de mayor protagonismo en la sección de internacional no suelen coincidir con los de mayor protagonismo de los medios dominantes. En los medios de la inmigración no son los Estados Unidos ni los 
países ricos de Europa los estados que más noticias internacionales ofrecen a estos medios sino que son los países a los que pertenecen los receptores; los grandes protagonistas de la información internacional. De hecho la sección de Internacional se rotula en Latino con el nombre de "Mi país" y en Sí, se puede con el de "Los países de origen".

Así pues, las noticias de ámbito internacional, con independencia de la sección en la que se encuentren, se refieren sobre todo a los países de origen de los inmigrantes y especialmente hay una clara presencia de los países latinoamericanos. El 24,5\% de las informaciones de estos dos semanarios tiene su origen en países de Latinoamérica.

En este punto conviene hacer una aclaración. Latino y Sí, se puede son dos semanarios que compiten prácticamente por un mismo público, aunque como punto de partida debemos recordar que, en este aspecto, presentan pequeñas diferencias. Latino se dirige a la comunidad hispanoamericana únicamente y Sí, se puede amplió algo más su público potencial, al dirigirse a toda la comunidad inmigrante. De hecho, la línea editorial de Sí, se puede, reforzando este aspecto, señala no tener preferencias por ningún grupo de inmigrantes pero lo cierto es que Sí, se puede presta sobre todo atención a la comunidad latina. Al menos las cifras así lo demuestran.

Junto a una información más próxima a sus valores culturales y su comunidad de origen el periodismo de servicio es otro rasgo que caracteriza a estas publicaciones. El análisis efectuado pone de manifiesto que estas publicaciones buscan la utilidad en los contenidos. Y es que tres de cada nueve textos analizados presentan rasgos propios del periodismo de servicio. En la mayoría de los casos se trata de una información movilizadora que le propone al lector actividades u ofertas culturales varias y le indica cómo y en qué horario puede acudir a ellas. Igualmente es frecuente en las páginas de estos semanarios la presencia de un consultorio de abogados a los que el lector puede formular personalmente las dudas que tenga sobre materia legislativa. También resultan habituales las informaciones sobre cursos, sobre todo cursos gratuitos, que se ofrecen a toda la comunidad o específicamente a la comunidad inmigrante. En definitiva, no resulta extraño encontrar en estas publicaciones textos informativos que recomienden o aconsejen actividades a sus lectores. 
Tabla II. Temática de la información de servicio

\begin{tabular}{|lccc|}
\hline & Latino & Sí, se puede & Total \\
\hline Información movilizadora & 55,6 & 24,4 & 45 \\
\hline Consejos- recomendaciones & 10,7 & 11,1 & 10,8 \\
\hline Información de cursos & 5,7 & 5,6 & 5,6 \\
\hline Consultorio & 5,7 & 4,4 & 5,2 \\
\hline Otros & 22,3 & 54,5 & 33,4 \\
\hline & 100 & 100 & 100 \\
\hline
\end{tabular}

Fuente propia

\subsubsection{Trasfondo migratorio}

El deporte junto con la política son los temas a los que más atención suelen prestar estos semanarios, aunque, en cualquier caso, lo cierto es que la temática de estas publicaciones es, como en cualquier otra publicación de contenido generalista, muy variada. Ahora bien, a pesar de la diversidad temática, un porcentaje muy considerable de esas informaciones presenta un trasfondo temático migratorio. Una de cada cuatro noticias que aparecen en estos diarios aborda cuestiones relacionadas con la inmigración. Al inmigrante se le dibuja como una persona que contribuye económicamente a la buena marcha del país y es que el 12,6\% de las informaciones que tocan la cuestión de la inmigración lo hacen para destacar este aspecto positivo del fenómeno de la inmigración. Igualmente se dibuja al inmigrante como una persona que participa de las celebraciones populares o tradicionales originarias de su tierra que ahora se han trasladado con ellos al país de acogida. 
Tabla III. Aspectos de la inmigración presentes

\begin{tabular}{|lccc|}
\hline & Latino & Sí, se puede & Total \\
\hline Tramitación de documentos, regularización & 14,2 & 10,9 & 12,6 \\
\hline Contribución económica, consumo & 8,5 & 17,5 & 12,6 \\
\hline Descripción de la experiencia migratoria & 17 & 1,1 & 9,6 \\
\hline Celebraciones populares & 12,3 & 6,5 & 9,6 \\
\hline Inmigrantes como víctimas & 12,3 & 3,3 & 8,1 \\
\hline Debate política española /Gestión de fronteras & 2,7 & 9,7 & 6 \\
\hline Entrada irregular / expulsiones & 1,9 & 8,7 & 5,0 \\
\hline Inmigrantes como delincuentes & 2,8 & 0 & 1,5 \\
\hline Otros & 28,3 & 42,3 & 34,8 \\
\hline Total & 100 & 100 & 100 \\
\hline
\end{tabular}

Fuente propia

En escasas ocasiones al inmigrante se le perfila bajo valores negativos, como la delincuencia o la violencia. El inmigrante aparece en estas publicaciones como víctima en el 8,1\% de los relatos que abordan aspectos relacionados con la inmigración. Aunque las informaciones que analizan el fenómeno de la inmigración no suelen tener connotaciones negativas hay ejemplos claros donde la información que se ofrece no dibuja una realidad deseada. La existencia de discriminación, de racismo, es una realidad que los medios de la inmigración aunque no acostumbran a mostrar no pueden obviar si pretenden ser un reflejo de la realidad migratoria donde las luces y las sombras abundan.

Al margen de esta crítica creemos que en estas publicaciones lo más positivo es que la propia comunidad inmigrante participa activamente en el proceso de auto-representación, ofreciendo a los lectores de estas publicaciones una imagen que difiere mucho de la habitual en los medios dominantes. Estas publicaciones satisfacen un deseo del colectivo inmigrante, tener voz en los medios. Y es que los medios tradicionales no suelen dibujarles como “sujetos con voz propia y personalidad definida” (Sánchez: 2005). Así ellos, en estas publicaciones, suelen ser los protagonistas, las fuentes de las informaciones y, además, los periodistas que elaboran esas informaciones. Estos medios constituyen una alternativa comunicativa, pues, para el colectivo inmigrante en España: un espejo de su realidad migratoria, un altavoz para sus reivindicaciones y problemáticas y una ayuda para la integración. 
Ahora bien, en este último aspecto es necesario resaltar la existencia de posiciones dispares. Si bien consideramos que estos medios pueden ayudar al inmigrante a adaptarse a la sociedad de acogida, algunos autores recuerdan que estos medios pueden también contrarrestar la asimilación cultural (Riggins, 1992).

\section{Publicidad}

En la muestra de 221 anuncios obtenidos del vaciado de los diarios (de enero a marzo de 2008) hemos identificado en cada uno de ellos las siguientes variables: anunciante, marca, sector, sector secundario, target y posibles patrocinadores de dicha muestra. Y nos hemos centramos en la variable sector elaborando 12 categorías diferentes de productos y servicios: alimentación, automoción, bebidas, construcción, cultural, deportes, distribución y restaurantes, energía, finanzas, hogar, industria, limpieza, servicios públicos y privados, transportes, viajes y turismo y ocio. Y de éstas, de acuerdo con el vaciado, hemos detectado subcategorías adicionales.

- Alimentación: distribución en destino de productos propios, distribución de productos en destino y distribución en origen de productos propios.

- $\quad$ Automoción: compra de coches

- $\quad$ Bebidas: refrescantes y alcohólicas

- Construcción: en origen y en destino

- Cultura: cursos de formación, universidad, revistas

- $\quad$ Deporte: fútbol

- Distribución y restauración: servicios de mensajerías, restaurantes de comida de origen, envío de dinero por entidades bancarias, préstamos, hipotecas y reunificación de deudas, bancos, microcréditos, tarjetas de créditos y domiciliación bancaria

- Hogar : Muebles y electrodomésticos

- $\quad$ Servicios públicos y privados: reparación e instalaciones, centros de belleza, salud, ofertas de trabajo, información del gobierno, servicios jurídicos, servicio de organización de eventos y servicio de limpieza.

- Transporte y turismo: líneas aéreas, agencias de viajes y turismo de las administraciones públicas 
- Ocio: concursos, espectáculos, discotecas, festivales y eventos, música, medios de comunicación y loterías.

- Otros: textil, quiromancia, informática, telefónica y publicidad electoral.

A partir de esta categorización hemos podido identificar las tipologías de productos y servicios más ofertados a este colectivo y analizar estas ofertas con su estilo de vida en nuestro país que va más allá de los estereotipos asociados con la inmigración. Los principales resultados obtenidos están vinculados con las categorías de los productos y servicios de los anunciantes que realizan más publicidad, el lenguaje utilizado en las piezas publicitarias y la representación de los colectivos inmigrantes en los anuncios.

Las categorías en las que los anunciantes realizan más publicidad son en el sector de servicios, distribución y restauración. Así mismo, resulta llamativo el número de anuncios de empresas de viviendas en destino y en origen. Parece que algunos inmigrantes tienen la intención de volver o de invertir su dinero en la adquisición de viviendas en origen. A un mismo tiempo, hay una oferta de viviendas en destino que muestra la intención de querer establecerse en España. Esto está vinculado con el número de anuncios de restauración de comida típica y como alrededor de este servicios proliferan otros servicios asociados con el servicio de limpieza y reparación de locales. Igualmente los anuncios de organización de fiestas para niños explican la agrupación familiar de estos colectivos.

Figuras 1 y 2. Anuncios del sector inmobiliario
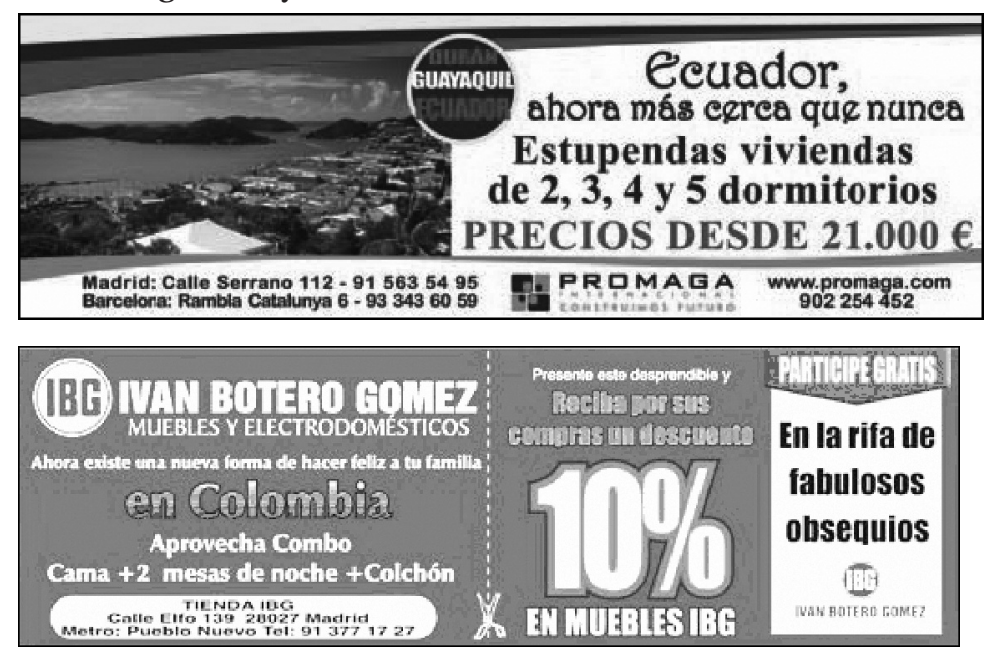

Fuente: Sí, se puede 15/03/08 
Otro dato importante que hemos identificado de este vaciado es el lenguaje publicitario que en pocas ocasiones se traduce al idioma de origen. Solamente se han encontrado dos anuncios, uno del Banco de Rumanía, y otro, una oferta de cursos traducido en distintos idiomas (en Toumai y en Sí, se puede). Este tipo de anuncios no son muy frecuentes aunque conviene recordar que dos de los medios analizados se dirigen a latinoamericanos y que el castellano nos une. De la muestra de anuncios obtenida se observa que no se está segmentando al colectivo. La publicidad va dirigida al colectivo de inmigrantes como si se tratase de una unidad. Hay excepciones como traducir un anuncio en distintos idiomas manteniendo el código visual, dirigir campañas puntuales a un colectivo específico o utilizar símbolos fácilmente reconocibles por un colectivo. Pero debemos seguir investigando para saber si en un futuro próximo habrá una nueva estrategia publicitaria o se mantendrá la tendencia actual.

Figura 3. Publicidad en idiomas propios

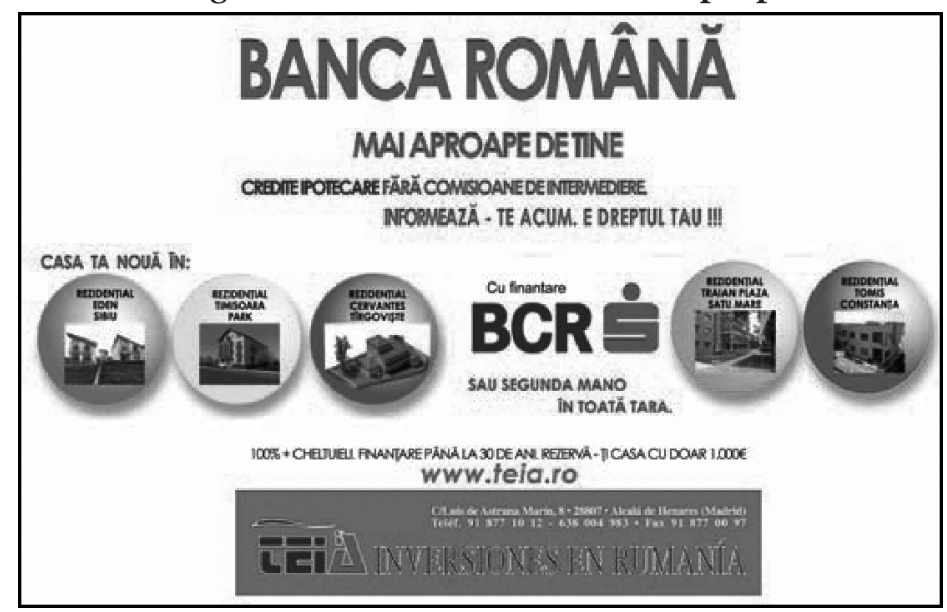

Fuente: Sí, se puede 08/03/08

Respecto a la representación de los inmigrantes, en publicidad cada vez más se evita utilizar estereotipos, aunque se siguen utilizando símbolos como las banderas o los rasgos físicos para que los inmigrantes se identifiquen con estas marcas y consigan fidelizarlos en los anuncios de empresas de telecomunicaciones, de informática, la organización de fiestas o conciertos, las compañías de envío de dinero y la banca. 
Figuras 4-7. Búsqueda de identificación en la publicidad
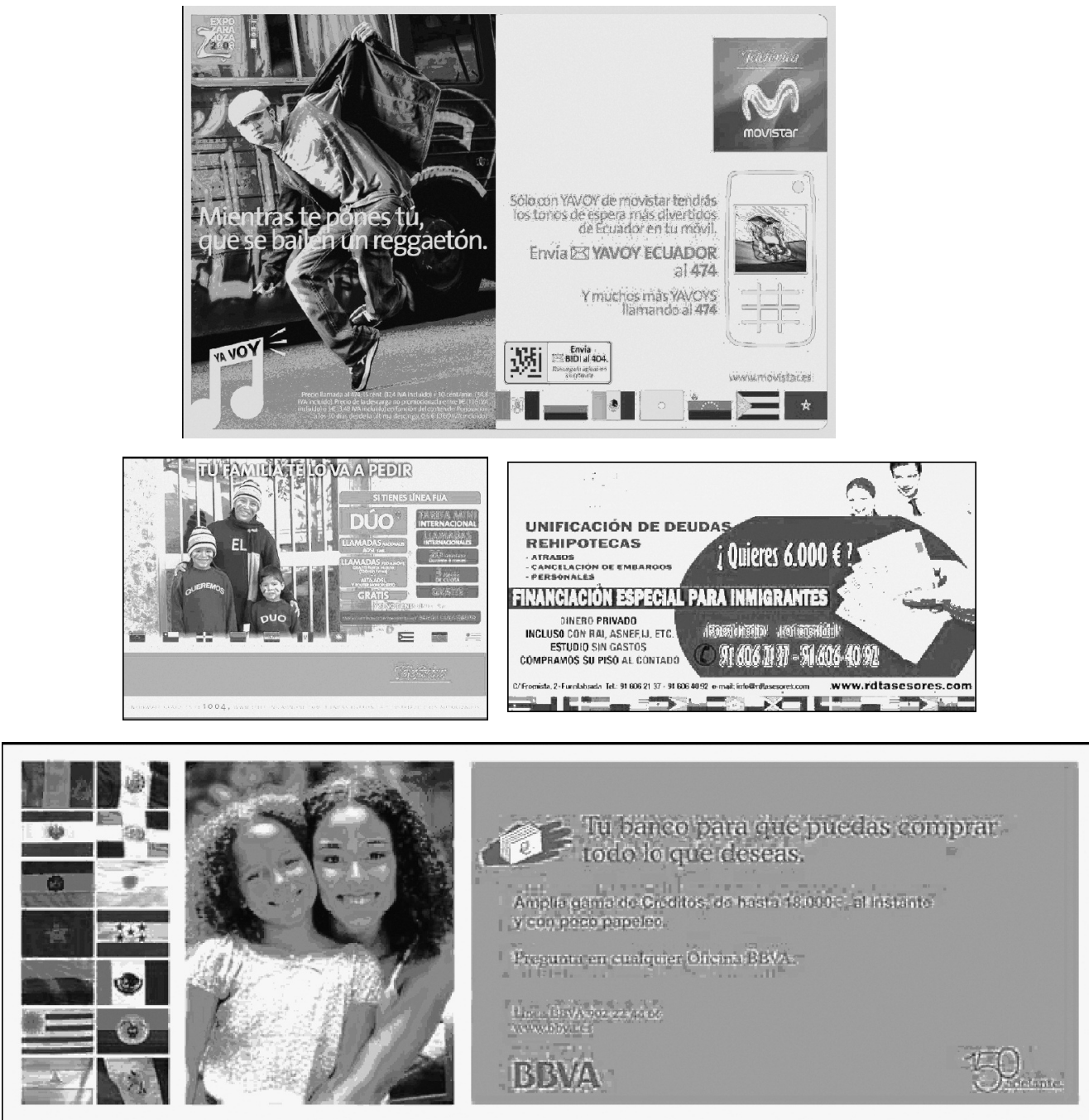

Fuente: Latino 14/03/08 y 19/01/08 
Éstas son algunas de las conclusiones a modo de radiografía que se obtiene de la categorización de los productos y servicios dirigidos a inmigrantes y que se debe seguir estudiando y analizando por su constante fluctuación en nuestra sociedad.

También llaman la atención determinados anuncios relativos a este colectivo que no se adecuan a los estilo de vida de la población autóctona como son los relativos a videntes, las oferta de trabajo y los de organización de fiestas infantiles. El número de anuncios relativos a la quiromancia son numerosos y entendemos que es fruto de su cultura, de hecho los videntes que aparecen en los anuncios son de origen africano o latino. En relación con los anuncios de trabajo son destacables tres ofertas de empleo que se repiten: gogós en discotecas, teleoperadores y militares para las Fuerzas Armadas. Se repiten asimismo en las publicaciones latinas los anuncios destinados a la organización de fiestas infantiles. Se entiende que esta comunidad vive en familia y que en su estilo de vida acostumbran a organizar eventos para los menores. Hábito que proviene de sus países de origen y de su proximidad a Estados Unidos.

\section{Conclusiones}

El análisis de los contenidos tanto informativos como publicitarios de estos medios nos lleva a concluir que los medios para los inmigrantes contribuyen a la cohesión cultural de sus receptores, les permiten recrear su propia cultura y sus valores y al mismo tiempo les permiten acceder a una información hecha a su medida, ajustada a sus necesidades y desde su propia visión. La información internacional se aborda desde una perspectiva distinta en la que ellos adquieren un mayor protagonismo. En asuntos de política internacional pueden ser los mismos hechos pero vistos siempre desde el prisma del inmigrante. Aunque en publicidad solo se ha identificado un anuncio electoral del PSOE, y no de otro partido previsiblemente, estos anuncios se incrementarán, pues se empieza a considerar al inmigrante como posible votante.

Estas publicaciones posiblemente satisfagan el deseo del colectivo inmigrante de tener voz en los medios pues los inmigrantes se constituyen en los protagonistas, en las fuentes de las informaciones e incluso en el periodista que elabora las informaciones que aparecen en estas publicaciones. Esta circunstancia favorece los procesos de identificación del público lector con la publicación y les facilita a los inmigrantes la posibilidad de recrear en esos medios su comunidad trasnacional. Y es que sus valores, sus creencias, sus ídolos musicales o sus equipos deportivos... aparecen siempre reflejados en las páginas de estas publicaciones. La representación de los valores se refleja también en publicidad en anuncios relativos a fiestas, restauración o actividades deportivas. 
La doble dimensión de este estudio sobre los contenidos de las publicaciones analizadas nos permite constatar que estas publicaciones no siempre se pliegan, como es la norma, al interés de sus anunciantes. El periodismo de servicio queda a veces por encima de los intereses publicitarios. Aunque los anuncios sobre préstamos fáciles son muy frecuentes en estas publicaciones, Latino advierte de los riesgos de esos créditos que se anuncian como la gran solución a los problemas económicos. Sus lectores son personas especialmente vulnerables que presentan dificultades económicas y que a veces está bien que la voz amiga de su semanario les advierta de los riesgos de los créditos rápidos. Bajo el título "Dinero fácil pero muy caro" (Latino, 22 de junio de 2007. no 114) Latino advertía así a sus lectores.

En publicidad las empresas financieras como La Caixa o Banesto son las que más se anuncian ofreciendo envíos de dinero, hipotecas y domiciliación de nóminas. Hay numerosos anunciantes que ofertan reunificación de deudas pero que no indican quiénes son y solamente indican un número de teléfono, hecho que según la LGP (Ley General de Publicidad) es ilegal porque no se identifica el anunciante. Entre los servicios de mensajería Money Gram y Western Union son los que invierten más en publicidad, seguido de la sociedad estatal de Correo y Telégrafos con anuncios a toda página y en color. También, al igual que abundan los consultorios jurídicos en estas publicaciones, los anuncios de servicios jurídicos son de los más numerosos porque la situación jurídica de los inmigrantes es complicada no sólo por los problemas en nuestro país en temas de nacionalidad, trabajo, homologación de títulos, sino por causas civiles de divorcio, relaciones paterno filiales, herencias y problemas financieros, tributarios o mercantiles que se ocasionan en su país y que deben resolverse en España.

El cruce de los resultados del estudio de los contenidos informativos y de la publicidad muestra que los mensajes se ajustan a los deseos y necesidades de los inmigrantes, en definitiva, de su público objetivo. Y que las noticias más relevantes en los diarios coinciden con los anuncios publicitarios. Esto demuestra que la información ofrecida a este colectivo se adecua a sus necesidades jurídicas, laborales y de ocio.

\section{Referencias bibliográficas}

Benitez, J.L. (2007): “Comunidad salvadoreña inmigrante en el área metropolitana de Washington D.C.: procesos de identidad colectiva y comunicación transnacional”, ECA Estudios Centroamericanos, vol. 699-700, pp. 15-40.

Franquet, R, y Ribes, F.X. (2004): “A ritmo latino. Los radiodifusores estadounidenses buscan empatizar con la audiencia hispana”, Telos, vol. 70, pp. 64-72.

Georgiou, M. (2006): Diasporic Transnationalism and Mediated Spatialities, UK: Leeds University. 
Gómez Mompart, J.L. (2008): “Diversidad latina y comunicación panhispana en la prensa en español de Nueva York al inicio del siglo XXI”, Doxa, vol. 6, pp. 15-51

Gómez-Escalonilla, G. (coord). (2008): Voces de la inmigración. Medios latinos en Madrid, Madrid: Universitas.

Granados, A. (2006): "Medios de comunicación, opinión y diversidad (social y cultural): reflexiones en torno al fenómeno migratorio" en Lario Bastida, M. (coord.): Medios de comunicación e inmigración, Murcia: Convivir sin racismo (CAM, Obra social). pp. 59-84, Disponible en Internet [URL] http://www.acsur.org/acsur/seccion/publicacion/Medios\%20e\%20inmigraci\%F3n.pdf

Holland, J. Gentry, J. (1999): "Ethnic Consumer Reaction to Targeted Marketing: A Theory of Intercultural Accommodation”, Journal of Advertising, Spring 1999, 28, 1.

Lee, E., Fairhurst, A.; Dillard, S. (2002): “Usefulness if Ethnicity in International Consumer Marketing”, Journal of International Consumer Marketing, vol. 14 (4).

López, X., (2007): “Un sector en auge. Evolución de la prensa en español en Estados Unidos”, Telos, vol. 70, pp. 45-97.

Lorite García, N. (2004): Cómo miran los medios la inmigración y transmiten la diversidad. Dialogo Comunicación y diversidad cultural In Com-UAB. Barcelona: Forum Universal de las Culturas. Disponible en Internet [URL] http://www.migracom.com/publicaciones/fichero_36.pdf

Martínez Pastor, E.: “Campañas publicitarias para ciudadanos inmigrantes”, en La publicidad se acerca a la Inmigración. Un factor de integración de la diversidad cultural, Madrid: Observatorio de las Realidades Sociales y la Comunicación.

Martínez Pastor, E. y Vizcaíno-Laorga, R. (2008): "Publicidad institucional como fenómeno integrador ante la inmigración en España: régimen jurídico", Revista Latina de Comunicación Social, vol. 63, pp. 90-98. Disponible en Internet [URL] http://www.ull.es/publicaciones/latina/_2008/10_Fuenlabrada /Vizcaino_y_Martinez.html

Prado, E. y Delgado, M. (2004): Televisión hispana en Estados Unidos. Tensiones económicas y cambios generacionales, Telos, vol. 70, pp. 45-97.

Retis, J. (2008): Espacios Mediáticos de la Inmigración en Madrid: Génesis y Evolución, Observatorio de las migraciones y de la convivencia intercultural de la ciudad de Madrid. Colección de monografías. No 4. Serie Azul: Estudios Generales.

Riggins, S. H., (ed.). (1992): Ethnic Minority Media: An International Perspective, London: Sage Publications.

Rios, D. y Mohamed. (Eds.) (2003): Brown \& Black communication: Latino and African American conflict and convergence in mass media. Westport, CT: Greenwood Press.

Rojas, V. (2004): “The gender of Latinidad: Latinas speak about Hispanic Televisión”. The Communication Review, vol. 7. pp. 125-153.

Sánchez, J. (2005): Medios de comunicación e inmigración. En aula intercultural. Disponible en Internet [URL] http://www.aulaintercultural.org/print.php3?id_article=748 
Suro, R. (2004): "Changing Channels and Crisscrossing Cultures: A Survey of Latinos on the News Media". Pew Hispanic Center. Disponible en Internet [URL] http://pewhispanic.org/files/reports/27.pdf

Torres, I. y Gelb, B. (2002): “Hispanic-targeted Advertising: More Sales?”, Journal of Advertising Research, Nov-Dic, NY, vol. 62 . 